\title{
Site-Specific Bio-Functionalization of Surfaces by Means of Metal Nanostructures
}

\author{
Tobias Peissker $^{\dagger}$, Barbara Gysbrechts, Gloria Fabris, Olivier Deschaume, \\ Danielle R. Rand, Kelly Houben, Christian P. Romero, Margriet J. Van Bael* and Carmen Bartic*
}

\begin{abstract}
We present an approach to control the immobilization process of biomolecules by functionalization of $\mathrm{SiO}_{2}$ substrates decorated with nanostructures. The creation of specific protein permissive and protein resistant areas leads to a directed and site-specific immobilization of proteins. We make use of molecular beam epitaxy and laser assisted cluster deposition to create Au nanostructures on PEG-silanized $\mathrm{SiO}_{2}$ substrates. The chemical contrast provided by the two materials, namely Au and PEG on the substrate surface supports the specificity of thiol-based Au functionalization for biomolecule attachment.
\end{abstract}

\section{INTRODUCTION}

The immobilization of biomolecules on solid substrates plays an important role in fundamental as well as applicationdriven research for biosensing, catalysis, tissue engineering, bioseparation and bioelectronics. The immobilization approach strongly influences the structure of the molecule and finally its functionality. Since biomolecules are in general attached to surfaces different from their native environment, it remains challenging to preserve their biological activity. Nanotechnology offers the possibility to control the site-specific attachment of biomolecules with minimized contact between adjacent molecules. Surfacedeposited metallic nanostructures with sizes comparable to those of biomolecules are promising for achieving nanoscale control of protein attachment [1]. By providing specific binding sites for immobilization, biomolecules can be attached either by direct chemisorption or via linkerchemistry and bioaffinity immobilization. Inter-molecular interaction between bound molecules can be reduced by tuning the inter-particle spacing. However, the control of the immobilization at a low-density level requires a reduction of non-specific attachment of biomolecules on the substrate material. This is of high importance as silicon-based materials, e.g. silicon, silicon dioxide, silica and quartz, are frequently used as substrate material for the manufacturing of biosensors but show a substantial tendency for protein

This research has been supported by the Fund for Scientific Research Flanders (FWO-Vlaanderen), by the Belgian Interuniversity Attraction Poles (IAP) and the Flemish Concerted Action (GOA) research programs. Tobias Peissker acknowledges financial support from the Agency for Innovation by Science and Technology in Flanders (IWT). Kelly Houben is a PhD research fellow of the FWO-Vlaanderen.

T. Peissker, B. Gysbrechts, G. Fabris, O. Deschaume, K. Houben, C. P. Romero, M. J. Van Bael and C. Bartic are with the Laboratory of Solid State Physics and Magnetism, KU Leuven, Celestijnenlaan 200D, B-3001 Leuven, Belgium tobias.peissker at fys.kuleuven.be

T. Peissker, D. R. Rand and C. Bartic are with imec, Kapeldreef 75, B-3001 Leuven, Belgium

* equally contributing authors

$\dagger$ corresponding author adsorption and hence bio-fouling [2]. The limitation of non-specific protein adsorption on the substrate material is therefore crucial [3]. A promising way to circumvent this problem is the grafting of a polyethylene(glycol) (PEG) layer onto the substrate. A silane-modified PEG (PEG-silane) layer was shown to reduce the amount of non-specifically adsorbed protein on $\mathrm{SiO}_{2}$ [4]. However, for a nano-structured system it needs to be taken into account, that a $\mathrm{PEG}_{6 / 9}$ molecule is $2-3 \mathrm{~nm}$ long [5] and hence might be in the same size range as the deposited nanostructures. As the polymer chains are highly flexible, one would expect that PEG-silane molecules cover or shield neighboring nanostructures and limit or hinder their biofunctionalization. The increase of the nanostructures size to values much higher then the polymer chain is one solution [8]. In this paper, we describe an approach to deposit the metallic nanostructures directly on the protein-repellent layer, thus reducing the amount of protein non-specifically attached to the background while presenting specific binding sites. Such a two-material system exploits the chemical contrast between the substrate and the cluster material. To investigate these nanometer-scale systems we have used atomic force microscopy (AFM), which, in combination with contact angle measurements (CA) and quartz crystal microbalance (QCM), yields direct information on the surface morphology and chemistry.

\section{MATERIALS AND METHODS}

We used thermally oxidized $(300 \mathrm{~nm})$ p-doped Si wafers $\left(\mathrm{SiO}_{2}\right)$, cleaned in Piranha solution $\left(3: 1 \quad \mathrm{H}_{2} \mathrm{SO}_{4}: \mathrm{H}_{2} \mathrm{O}_{2}\right.$ at $100^{\circ} \mathrm{C}, 15 \mathrm{~min}$ ) and $\mathrm{APM}$ (Standard clean 1, 4:1:1 DIW: $\mathrm{NH}_{4} \mathrm{OH}: \mathrm{H}_{2} \mathrm{O}_{2}$ at $\left.70^{\circ} \mathrm{C}, 15 \mathrm{~min}\right)$. QCM crystals (QSX301 and QSX303, Q-Sense, Sweden) were cleaned in acetone, isopropyl alcohol (each $5 \mathrm{~min}$ ) and $\mathrm{UV} / \mathrm{O}_{3}$ (15 min). Immediately after the cleaning, the samples were transferred to their respective incubation solution. For the protein-repellent layer, PEG-silane $\left(10 \mathrm{mM} \mathrm{PEG}_{6 / 9}\right.$ $\left.\left(\left(\mathrm{CH}_{3}\right)(\mathrm{PEG})_{n}\left(\mathrm{CH}_{2}\right)_{3} \mathrm{OSi}\left(\mathrm{OCH}_{3}\right)_{3}, \mathrm{n}=6-9\right)\right)$ was grafted to cleaned $\mathrm{SiO}_{2}$ in water free toluene for $18 \mathrm{~h}$, followed by rinsing with toluene, acetone, DIW and methanol and a short annealing under inert atmosphere at $110^{\circ} \mathrm{C}$. The thiol-linker molecules $\left(\mathrm{HS}\left(\mathrm{CH}_{2}\right)_{11}(\mathrm{PEG})_{6}\right.$-biotin) were attached in ethanol for $3 \mathrm{~h}$, followed by rinsing with ethanol. MBE Au nanostructures (high and low coverage) were deposited in situ by evaporation from a Knudsen cell at a 
deposition rate of 0.64 and $0.01 \AA / s$, respectively.

\section{RESULTS AND DISCUSSION}

\section{A. Grafting of a protein-repellent layer onto $\mathrm{SiO}_{2}$ substrates}

After grafting PEG-silane onto clean $\mathrm{Si} / \mathrm{SiO}_{2}$ substrates, the static contact angle of water (CA) increased from $11 \pm 3^{\circ}$ to $38 \pm 2^{\circ}$, comparable to previously reported values [6]. This decrease in hydrophilicity can be attributed to the composition of the PEG-silane polymer, namely a combination of a hydrophobic terminal group (R$\left.\mathrm{CH}_{3}\right)$ and the hydrophilic backbone $\left(\left(\mathrm{CH}_{2} \mathrm{CH}_{2} \mathrm{O}\right)_{n}\right)$. The incorporation of water into the backbone plays an important role in the protein-repellent properties of the film [10] The rms roughness, determined by analysis [7] of AFM topography images, slightly increased and the grafted film appeared to a large extent flat and uniform (Fig. 1a and b). XPS analysis (data not shown) of the $\mathrm{C} 1 \mathrm{~s}$ spectra
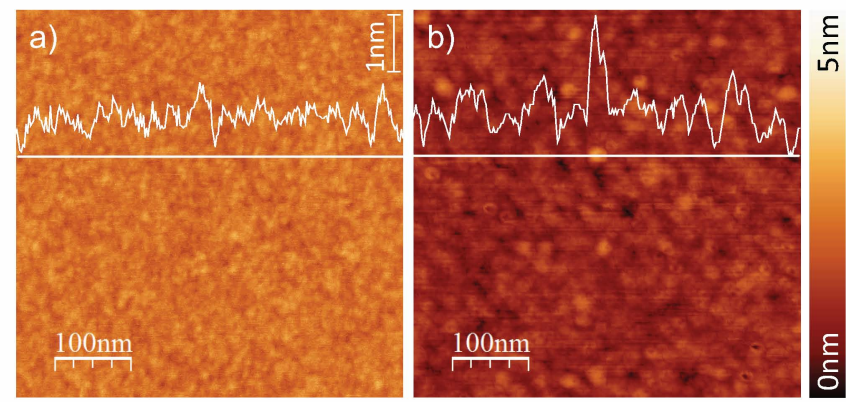

Fig. 1. AFM topography images of a) clean $\mathrm{SiO}_{2}$ and b) of a $\mathrm{SiO}_{2} / \mathrm{PEG}$ silane substrate. The insets show the line profile of the corresponding lines. A vertical scale bar $(1 \mathrm{~nm})$ is included next to the cross section in a) and is the same for both cross sections.

showed the $\mathrm{C}-\mathrm{O}$ and the C-C/C-H peaks at $283.5 \mathrm{eV}$ and $285 \mathrm{eV}$, respectively, characteristic for PEG moieties [2]. Force spectroscopy (data not shown) in air reveals an increase in adhesion between the $\mathrm{SiO}_{2}$-tip and the $\mathrm{SiO}_{2}$ sample after PEG-silanization by over $200 \%$. This increase in adhesion can be attributed to the highly hydrated PEG-silane layer attached to surface. To evaluate the protein-repellent properties of the PEG-silane layer we quantified the molecule adsorption on the PEG-silane. A quartz crystal microbalance $(\mathrm{QCM})$ relates the chnage in the resonance frquency of a piezoelectric crystal to the mass adsorbed on the crystal surface, i.e. the amount of adsorbed molecules, by the Sauerbrey equation [9]. The non-specific absorbtion of human $\mathrm{IgG}$ to $\mathrm{SiO}_{2} / \mathrm{PEG}$-silane covered quartz crystals was evaluated. QCM data revealed a reduction of non-specific adsorption of IgG on a PEG-silane surface was by approximately $95 \%$ (Tab. I), resulting in a protein coverage of less than $10 \%$ of the crystal surface. Non-specific attachment of IgG on clean $\mathrm{SiO}_{2}$ substrates led to a multi-layer coverage of $\mathrm{IgG}$, calculated based on the mass of one streptavidin molecule and its projected area (2.49E-10 ng and $58.5 \mathrm{~nm}^{2}$, respectively) and assuming the molecules forms a dense, flat layer on the crystal.
TABLE I

ADSORBED MASS AND CORRESPONDING PROTEIN COVERAGE ON THE QCM CRYSTALS AFTER INCUBATION WITH HUMAN IGG. ACCURACY OF THE DETERMINATION OF THE FREQUENCY IS $1 \mathrm{~Hz}$, RESULTING IN AN UNCERTAINTY OF THE ADSORBED MASS OF $\pm 3 \mathrm{NG} / \mathrm{CM}^{2}$.

\begin{tabular}{|c|c|c|c|}
\hline $\begin{array}{l}\text { Crystal } \\
\text { surface }\end{array}$ & $\begin{array}{r}\text { Adsorbed } \\
\text { mass of } \mathrm{IgG} \\
\left(\mathrm{ng} / \mathrm{cm}^{2}\right)\end{array}$ & $\begin{array}{r}\text { Number of } \\
\text { IgG molecules } \\
\left(1 / \mathrm{cm}^{2}\right)\end{array}$ & $\begin{array}{r}\text { Coverage } \\
\mathrm{IgG} \\
\text { (monolayer) }\end{array}$ \\
\hline $\mathrm{SiO}_{2}$ & 860 & $3.5 \mathrm{E} 12$ & 2.0 \\
\hline $\mathrm{SiO}_{2} / \mathrm{PEG}$-silane & 38 & $1.5 \mathrm{E} 11$ & 0.1 \\
\hline $\mathrm{Au}$ & 814 & $3.3 \mathrm{E} 12$ & 1.9 \\
\hline Au/PEG-silane & 422 & $1.7 \mathrm{E} 12$ & 1.0 \\
\hline
\end{tabular}

The attachment of PEG-silanes on polycrystalline Au surfaces was investigated by AFM and CA analysis. The CA decreased from $83 \pm 3^{\circ}$ to $64 \pm 3^{\circ}$ for Au and Au/PEGsilane surfaces, respectively. The increase in hydrophilicity suggests the attachment of the hydrophilic PEG-silane molecules on the substrate surface. Analysis of the morphology of the Au surface after PEG-silane attachment confirms, that PEG-silanes are attached to Au and reveals that those molecules agglomerate on the surface. As a result, the rms roughness increased stronger compared to PEG-silane on $\mathrm{SiO}_{2}$ from $1.0 \pm 0.2 \mathrm{~nm}$ (scan size $1 \times 1 \mu \mathrm{m}^{2}$ ) to $1.4 \pm 0.2 \mathrm{~nm}$, respectively. The non-specific adsorption of $\mathrm{IgG}$ molecules on silanized $\mathrm{Au}$ surfaces (Au/PEG-silane) was further investigated by QCM analysis (Tab. I) and shows a decrease in non-specific attachment of $\operatorname{IgG}$ on the Au/PEG-silane crystal surface compared to a clean $\mathrm{Au}$ surface. This implies that the PEG-silane layer is not effectively removed from the Au surface and hence prohibits the adsorption of $\operatorname{IgG}$ to the same extent as for a clean $\mathrm{Au}$ surface. Based on those considerations we conclude that the very flexible PEG-silane polymers might also cover deposited nanostructures or attach non-specifically to them, rendering them inaccessible for further functionalization. This is especially important for nanostructures in the size range of only a few $\mathrm{nm}$ as the length of a PEG-silane molecule is of a comparable size and the final thickness of the PEG-silane layer might even be larger depending on the grafting time [2].

\section{B. Functionalization of Au nanostructures on a PEG-silane layer}

Based on the above mentioned considerations, we have firstly grafted the protein-repellent PEG-silane onto the $\mathrm{SiO}_{2}$ surface, followed by the deposition of the $\mathrm{Au}$ nanostructures. We have deposited Au nanoclusters, preformed in a laser vaporization cluster source [11], in situ on PEG-silane covered samples. An abundance spectrum of the Au nanoclusters recorded before the deposition by a time-of-flight (TOF) mass spectrometer is shown in Fig. 2a. The size distribution of the detected clusters obtained from the TOF mass spectrum assumes a spherical shape for the clusters. Please note that neutral clusters are not detected 
in the mass spectrum but are deposited on the sample. The AFM topography image of nanoclusters deposited on
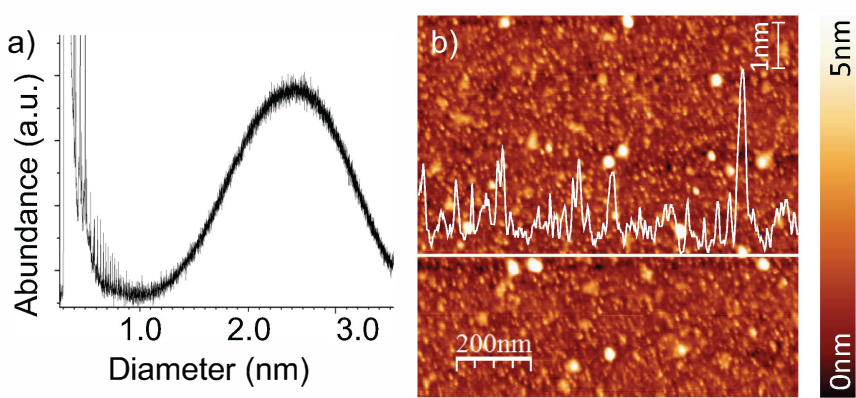

Fig. 2. a) Mass spectrum of non-mass selected, charged Au nanoclusters produced by the cluster deposition apparatus [11]. b) AFM topography image of non-mass selected $\mathrm{Au}$ nanoclusters, deposited on a $\mathrm{SiO}_{2} / \mathrm{PEG}$ silane sample, corresponding to the abundance spectrum shown in a). A vertical scale bar $(1 \mathrm{~nm})$ is included for the cross section in b).

a $\mathrm{SiO}_{2} / \mathrm{PEG}$-silane sample is shown in Fig. 2b. Next we deposited $\mathrm{Au}$ nanoclusters with a density of approximately 1680 clusters $/ \mu \mathrm{m}^{2}$ on a $\mathrm{SiO}_{2} /$ PEG-silane QCM crystal directly after the deposition on a $\mathrm{SiO}_{2}$ reference sample. We used the reference sample to exactly determine the coverage of nanoclusters on the sample surface. We used biotinylated thiols as linker molecule for the bioaffinity immobilization of streptavidin (see insets in Fig. 3). The streptavidin was successfully immobilized on this $\mathrm{SiO}_{2} / \mathrm{PEG}$-silane/Au/biotin-complex as can be seen in Fig. 3. After the introduction of streptavidin-buffer solution, an irreversible shift in frequency occurs, which indicates the attachment of streptavidin onto the crystal. The amount of adsorbed protein calculated based on the frequency shift was $129 \mathrm{ng} / \mathrm{cm}^{2}$, corresponding to a protein-coverage of the sensor surface of $0.46 \pm 0.02$ monolayer. We used molecular beam epitaxy (MBE) to deposit $\mathrm{Au}$ nanostructures with different surface coverage onto $\mathrm{SiO}_{2} / \mathrm{PEG}$-silane surfaces. The topography of $\mathrm{SiO}_{2} / \mathrm{PEG}$-silane samples with $1.0,0.5$, 0.4 and 0.2 monolayer Au coverage is shown in Figs. 4a-d, respectively. It can be inferred from these images that the density of the coverage increases from low to high densities. However, at very high coverage $(\geq 0.5$ monolayer), the nanostructure-density is too high in view of the framework of laterally distance-controlled biomolecule attachment. Using lower Au coverage, namely 0.2 and 0.4 monolayer (Figs. 4c, d), respectively, the density of the nanostructures is sufficiently low. We used a biotinylated thiol as linker molecule to immobilize streptavidin to the MBE-Au

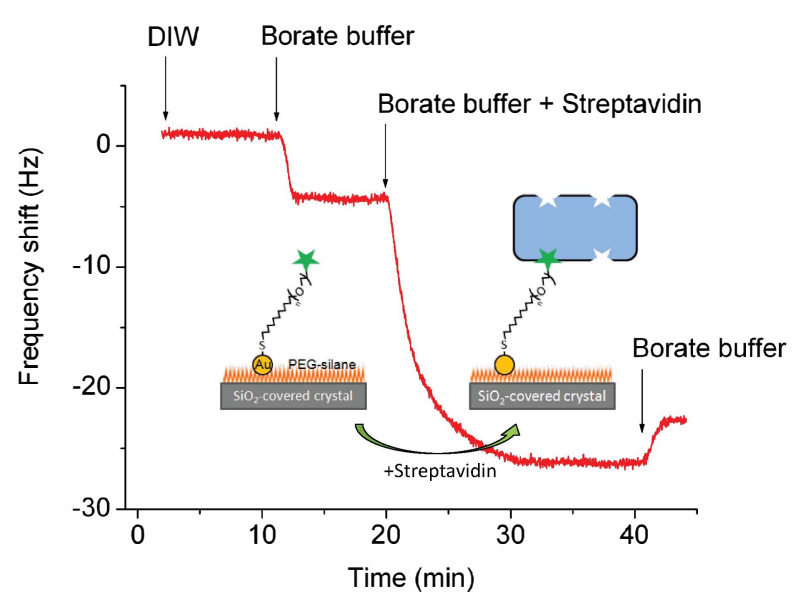

Fig. 3. QCM analysis of streptavidin adsorption. QCM crystal covered with $\mathrm{a} \mathrm{SiO}_{2} / \mathrm{PEG}$-silane/Au/biotin-complex. The shift of the resonance frequency of the QCM crystal indicates the adsorption of proteins to the complex.

nanostructures. The linker molecule was attached via thiol-binding to the Au. The immobilization of the linkerbiotin-streptavidin-complex increased the rms roughness from 0.5 to $1.2 \mathrm{~nm}$ (based on $1 \mathrm{x} 1 \mu \mathrm{m}^{2}$ topography images) and the size of the topographical features on the sample, as can be observed in Fig. $4 \mathrm{e}$ and $\mathrm{f}$, for 0.2 and 0.4 monolayer, respectively. The height of these features observed in the AFM images cannot be correlated with the expected size of a streptavidin molecule (approximated size $5 \times 4.5 \times 4.5 \mathrm{~nm}^{3}$, [12]). This might be attributed to the fact that the streptavidin molecule appears much smaller than its actual size in topographical images due to compressive force applied by the AFM tip [12]. The AFM imaging conditions during our experiment, namely intermittent contact mode in ambient air, leads to a dehydration of the molecule and might lead subsequently to a conformational change. However, supported by the results of the QCM experiments we can conclude that streptavidin is immobilized on the $\mathrm{Au}$ nanostructures. The large increase in lateral feature size can be rather attributed to a bending of the flexible linker backbone (as it is not supported by other molecules as for example in a self assembled monolayer of thiols) with a large, rather heavy streptavidin molecule attached to it. However, the results indicate a local attachment of streptavidin onto the nanostructures deposited on the surface. 

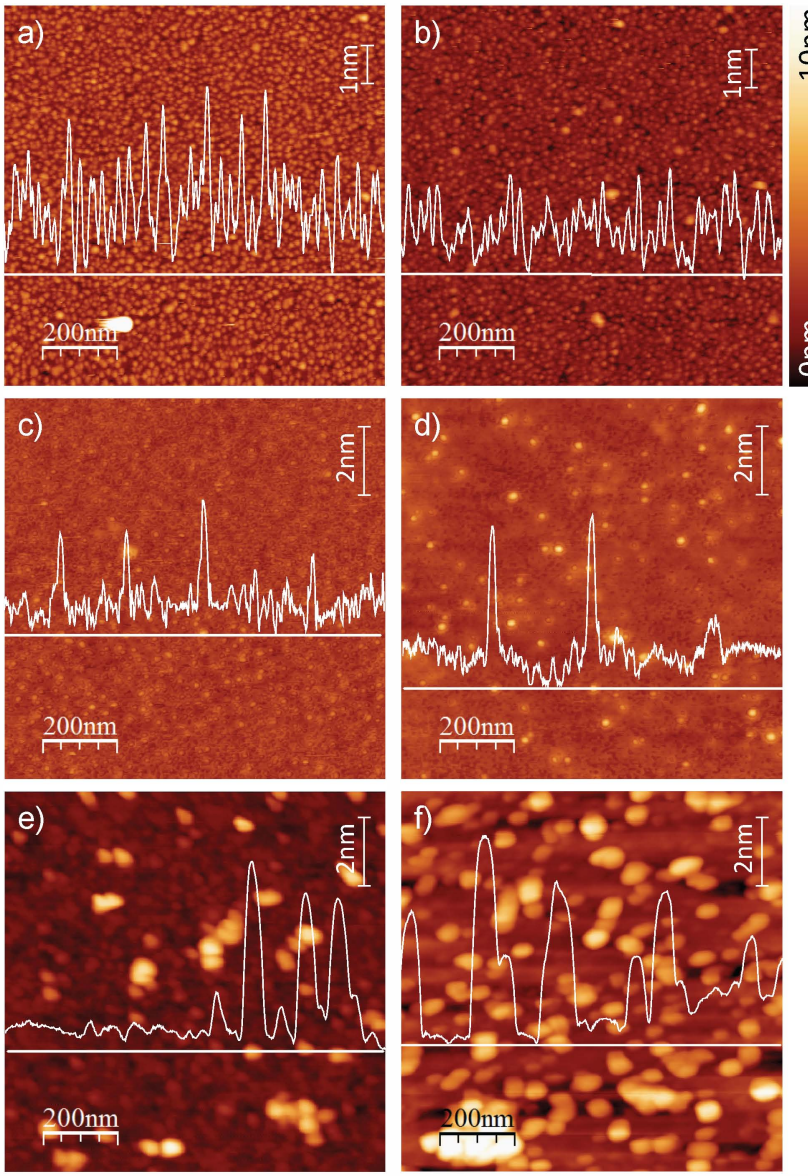

Fig. 4. AFM topography images of Au nanostructures, deposited on $\mathrm{SiO}_{2} / \mathrm{PEG}$-silane by MBE in different densities, namely a) 1.0 , b) 0.5 , c) 0.4 and d) 0.2 monolayer. Images e) and f) show the respective sample c) and d) after the attachment of the biotin-linker molecule and its functionalization with streptavidin. A vertical scale bar is included next to the respective cross section.

\section{SUMMARY AND CONCLUSIONS}

We have shown an alternative method to create nanometer sized structures for a chemical contrast with controlled density by sub-monolayer deposition of $\mathrm{Au}$ onto $\mathrm{SiO}_{2} / \mathrm{PEG}-$ silane surfaces. The PEG-silanes grafted to $\mathrm{SiO}_{2}$ substrates, form an effective protein-repellent layer on which we deposited $\mathrm{Au}$ nanostructures by vacuum techniques, namely by $\mathrm{MBE}$ and by preformed clusters from a laser vaporization cluster source. QCM analysis of streptavidin immobilization on $\mathrm{SiO}_{2} / \mathrm{PEG}$-silane/Au-biotin-complexes shows the successful attachment of the streptavidin to the $\mathrm{Au}$ nanostructures. AFM topography images reveal an increase in lateral dimensions after the streptavidin immobilization. In conclusion, we have created metallic nanostructures on a protein-repellent background which allows for the sitespecific functionalization of the nanostructures.

\section{ACKNOWLEDGMENT}

The authors wish to thank Dr. Karolien Jans and Tim Steylaerts (imec, Kapeldreef 75, B-3001 Leuven, Belgium) for their help with the surface chemistry and Dr. Marius Dobromir (Universitatea Alexandru Ioan Cuza din Iasi, Iasi, Romania) for the XPS measurements.

\section{REFERENCES}

[1] R.-E. Palmer, and C. Leung, "Immobilisation of proteins by atomic clusters on surfaces", TRENDS in Biotechnology, vol. 25, no.2, pp. 48-55, December 2006.

[2] S. Sharma, R. W. Johnson, and T. A. Desai, "XPS and AFM analysis of antifouling PEG interfaces for microfabricated silicon biosensors", J Biomed Mater Res., vol. 5, no.51(3), pp. 227-239, May 2004.

[3] N. A. Alcantar, E. S. Aydil, and J. N. Israelachvili, "Polyethylene glycol-coated biocompatible surfaces", Biosensors and Bioelectronics, vol. 20, no.2, pp. 343-351, September 2000.

[4] A. Papra, N. Gadegaard, and N. B. Larsen, "Characterization of Ultrathin Poly(ethylene glycol) Monolayers on Silicon Substrates", Langmuir, vol. 17, pp. 1457-1460, February 2001.

[5] I. Szleifer, "Protein Adsorption on Surfaces with Grafted Polymers: A Theoretical Approach", Biophysical Journal, vol. 72, pp. 595-612, February 1997.

[6] D. Janssen, R. De Palma, S. Verlaak, P. Heremans, and W. Dehaen, "Static solvent contact angle measurements, surface free energy and wettability determination of various self-assembled monolayers on silicon dioxide", Thin Solid Films, vol. 515, pp. 14331438, May 2006.

[7] I. Horcas, R. Fernandez, J.M. Gomez-Rodriguez, J. Colchero, J. Gomez-Herrero, and A.M. Baro, "WSXM: A software for scanning probe microscopy and a tool for nanotechnology", Review of Scientific Instruments, vol. 78, pp. 013705, January 2007.

[8] J. Blümmel, N. Perschmann, D. Aydin, J. Drinjakovic, T. Surrey, M. Lopez-Garcia, H. Kessler, and J. P. Spatz, ”Protein repellent properties of covalently attached PEG coatings on nanostructured $\mathrm{SiO}_{2}$-based interfaces", Biomaterials, vol. 28, no. 32, pp. 4739-4747, August 2007.

[9] G. Sauerbrey, "Verwendung von Schwingquarzen zur Wägung Dünner Schichten und zur Mikrowägung.", Zeitschrift für Physik, vol. 155, nr.2, pp. 206-222, February 1959.

[10] M. Heuberger, T. Drobek, and J. Vörös, "About the Role of Water in Surface-Grafted Poly(ethylene glycol) Layers.”, Langmuir, vol. 20, nr.22, pp. 9445-9448, September 2004.

[11] N. Vandamme, E. Janssens, F. Vanhoutte, P. Lievens, and C. Van Haesendonck, "Scanning probe microscopy investigation of gold clusters deposited on atomically flat substrates.", J. Phys.: Condens. Matter, vol. 15 , pp. 298, October 2003.

[12] A. L. Weisenhorn, F. J. Schmitt, W. Knoll, and P. K. Hansma, "Streptavidin binding observed with an atomic force microscope.", Ultramicroscopy, vol. 42-44, pp. 1125-1132, July 1992. 